\title{
Behavioral Healthcare Providers' Experiences Related to use of Telehealth as a Result of the COVID-19 Pandemic: An Exploratory Study
}

\author{
Abby Kisicki ( $\square$ kisicki@wisc.edu ) \\ University of Wisconsin-Madison \\ Sara Becker \\ Brown University \\ Michael Chaple \\ Columbia University \\ David H. Gustafson \\ University of Wisconsin-Madison \\ Bryan Hartzler \\ University of Washington \\ Nora Jacobson \\ University of Wisconsin-Madison \\ Ann A. Murphy \\ Rutgers, The State University of New Jersey \\ Stephanie Tapscott \\ Emory University

\section{Todd Molfenter} \\ University of Wisconsin-Madison
}

\section{Research Article}

Keywords: telehealth, virtual services, mental health, substance use disorder, reimbursement, qualitative research

Posted Date: November 23rd, 2021

DOI: https://doi.org/10.21203/rs.3.rs-965869/v1

License: (1) (1) This work is licensed under a Creative Commons Attribution 4.0 International License.

Read Full License 


\section{Abstract}

Background: Due to the COVID-19 pandemic, healthcare providers were forced to shift many services quickly from in-person to virtual, including substance use disorder (SUD) and mental health (MH) treatment services. This led to a sharp increase in use of telehealth services, with health systems seeing patients virtually at hundreds of times the rate as before the onset of the COVID-19 pandemic.

By analyzing qualitative data about SUD and MH care providers' experiences using telehealth, this study aims to elucidate emergent themes related to telehealth use by the front-line behavioral health workforce.

Methods: This study uses qualitative data from large-scale web surveys distributed to SUD and MH providers between May and August 2020. At the end of these surveys, the following question was posed in free-response form: "Is there anything else you would like to say about use of telehealth during or after the COVID-19 pandemic?" The 391 responses to this question were analyzed for emergent themes using a conventional approach to content analysis.

Results: Three major themes emerged in the data: COVID-specific experiences with telehealth, general experiences with telehealth, and recommendations to continue telehealth delivery. Convenience, access to new populations, and lack of commute were frequently cited advantages, while perceived ineffectiveness of and limited access to technology were frequently cited disadvantages. Also commonly mentioned was the relaxation of reimbursement regulations. Providers supported continuation of relaxed regulations, increased institutional support, and using a combination of telehealth and in-person care in their practices.

Conclusions: This study advanced our knowledge of how the behavioral health workforce experiences telehealth delivery. Further longitudinal research comparing treatment outcomes of those receiving inperson and virtual services will be necessary to undergird organizations' financial support, and perhaps also legislative support, of virtual SUD and MH services.

\section{Background}

The coronavirus (COVID-19) pandemic radically transformed mental health (MH) and substance use disorder (SUD) treatment. Due to social distancing requirements, stay-at-home orders, and other precautionary health measures, healthcare providers were forced to quickly shift many services from inperson to virtual, including SUD and MH treatment services [1, 2]. This led to a sharp increase in use of telehealth services, with health systems seeing patients virtually at hundreds of times the rate as before the onset of the COVID-19 pandemic [3]. Medicare, which in 2016 lagged in telehealth coverage compared to Medicaid and private insurers, has seen a significant increase in telehealth use, with $0.1 \%$ of Medicare primary care visits delivered virtually before the onset of the COVID-19 pandemic and nearly 50\% delivered virtually after March $2020[3,4]$. This spike in telehealth use occurred when SUD and MH treatment were already seeing steady increases in telehealth use. From 2010-2017, treatment via 
telehealth increased rapidly for both substance use and mental disorders [4], but not at the rates of growth experienced following the onset of COVID-19 safety procedures.

Regulatory changes that went into effect at the beginning of the COVID -19 pandemic facilitated the rapid and widespread expansion of telehealth. [5]. Depending on the state, these eased regulations include expanded reimbursement for Medicare, Medicaid, and private insurers; coverage parity or payment parity; provision of telehealth services across state lines; and reimbursement of telephone services at the same rate as in-person face-to-face visits [6]. It is unclear the extent to which these eased regulations will be permanently adopted once the COVID-19 public health emergency comes to an end, leaving providers uncertain in their planning for next steps for telehealth.

Research has shown that many providers indeed hope to continue the expanded use of telehealth after the COVID-19 pandemic. In their 2021 survey of MH care providers around the U.S., Guinart et al. [7] reported that $64 \%$ of respondents would like to continue using telehealth treatment with at least $25 \%$ of their clients and that $53 \%$ of these providers would like to continue using telehealth with at least $50 \%$ of their clients. Reimbursement for telehealth has often been cited as a barrier by $\mathrm{MH}$ care $[8,9]$ and SUD treatment providers [4], even before the COVID-19 pandemic and despite legislative action to address the reimbursement barrier [10]. Although providers have necessarily been integrating telehealth into their practices since the beginning of the pandemic, our knowledge of United States providers' experiences and the context behind their plans of whether to continue telehealth use after the COVID-19 pandemic remain extremely limited. This study aims to advance knowledge by analyzing qualitative data, gathered from web surveys administered in summer of 2020 [1, 2], about SUD and MH care providers' experiences using telehealth. The overarching objective of this analysis is to elucidate emergent themes related to the utilization of telehealth by the front-line behavioral health workforce.

\section{Methods}

This study uses data from large-scale web surveys distributed by designated regional Addiction Technology Transfer Centers (ATTCs) and Mental Health Technology Transfer Centers (MHTTCs) to SUD and $\mathrm{MH}$ providers, respectively, between May and August 2020 [1, 2]. The surveys collected information on how these providers applied virtual services during COVID-19, as well as their experiences with telehealth use [1, 2]. Results from the original analysis of the surveys showed that the SUD and MH providers intended to continue delivering many video services and some telephonic services after the COVID-19 pandemic public health safety procedures subside. At the end of these surveys, the following was posed in free-response form: "Is there anything else you would like to say about use of telehealth during or after the COVID-19 pandemic?"

Out of the 948 total SUD and MH survey responses, 391 (41\% of) respondents responded to this question. These 391 respondents' organization setting, organization type, and job function were also pulled from the survey data. Organization settings included rural, small city, suburban, and urban; organization types included specialty treatment, health system, opioid treatment programs, recovery 
community, FQHC, and other; job functions included counselor, administrator, physician/prescriber, recovery coach, and other. Each of these three characteristics were self-identified by the respondent.

The 391 verbatim responses were analyzed using NVivo 12 qualitative analysis software. One independent coder analyzed the responses using a conventional approach to content analysis [11]. As a first step, a research team consisting of three individuals (psychologist, one qualitative methodologist, one implementation science scientist) reviewed all of the responses and devised a preliminary coding structure. The independent coder then reviewed the responses and assigned an initial set of preliminary codes. During this first coding pass, the independent coder reviewed codes in an iterative fashion and added more granular themes to the preliminary coding structure as needed. The complete list of themes, along with excerpts from the data, were then reviewed by the research team. In a second pass, the independent coder revisited the data to consolidate repetitive sub-codes and refine and finalize the set of themes.

\section{Results}

\section{Sample characteristics}

Of the 391 respondents, $59.3 \%$ were SUD providers, and $40.7 \%$ were $\mathrm{MH}$ providers. The sample consisted of $46.3 \%$ counselors, $45.0 \%$ administrators, $2.8 \%$ physicians/prescribers, $2.6 \%$ recovery coaches, and $3.3 \%$ other (Table 1). As respondents were answering on behalf of their organization, they were not asked to provide socio-demographic data. Respondents were predominantly in specialty care settings and represented a mix of urban, suburban, and rural areas.

\section{Table 1}

\section{Sample characteristics}


Frequency \%

\section{Organization setting}

\begin{tabular}{lll}
\hline Rural & 101 & 25.8 \\
\hline Small city & 74 & 18.9 \\
\hline Suburban & 75 & 19.2 \\
\hline Urban & 140 & 35.8 \\
\hline Blank & 1 & 0.0 \\
\hline
\end{tabular}

\section{Organization type}

\begin{tabular}{lll}
\hline Specialty treatment & 269 & 68.8 \\
\hline Health system & 58 & 14.8 \\
\hline Opioid treatment programs & 25 & 6.4 \\
\hline Recovery community & 18 & 4.6 \\
\hline FQHC & 17 & 4.4 \\
\hline Other & 4 & 1.0
\end{tabular}

\section{Respondent job function}

\begin{tabular}{lll}
\hline Counselor & 181.0 & 46.3 \\
\hline Administrator & 176.0 & 45.0 \\
\hline Physician/Prescriber & 11.0 & 2.8 \\
\hline Recovery coach & 10.0 & 2.6 \\
\hline Other & 13.0 & 3.3 \\
\hline
\end{tabular}

\section{Overview of qualitative emergent themes}

Overall, three major themes emerged in the data: COVID-specific experiences with telehealth, general experiences with telehealth, and recommendations to continue telehealth delivery. Each of these themes contained multiple sub-themes, as elaborated in the following sections. 


\section{COVID-specific telehealth themes}

Multiple providers expressed the view that their organizations would not have begun to use telehealth if not for the onset of the COVID-19 pandemic. Within those responses that specifically commented on the pandemic, three subthemes emerged pertaining to COVID-specific advantages of telehealth adoption: continued provision of services during the pandemic, the ability to adhere to safety and social distancing guidelines, and experiences related to having children at home. Another COVID-specific sub-theme was the easing of regulatory restrictions, which facilitated providers' adoption of telehealth.

\section{Continued provision of services during the pandemic}

Many providers appreciated that virtual services could be used in place of in-person treatment once stayat-home orders and social distancing requirements began. Responses conveyed a sense of relief that providers were able to continue providing services to clients during COVID-19. Some respondents felt that the substitution of telehealth for in-person treatment "saved lives," "kept our patients out of the ED and psychiatric hospital" and was an "incredible lifeline" to clients. One provider stated that increased accessibility over the telephone was beneficial for clients, writing that "for clients to be able to access help before going into a full-blown crisis is priceless." Respondents also expressed gratitude that telehealth allowed them, as individual providers and as a field, to continue performing their jobs. Several comments credited the ability to provide virtual services with having "saved my job" and having "literally saved our organization and likely our clients."

\section{Safety and social distancing}

Respondents reported satisfaction with how telehealth adhered to social distancing guidelines and allowed clients and providers alike to minimize exposure to COVID-19. Telehealth was reported to be particularly beneficial for providers and clients at high personal risk of severe illness from COVID-19.

\section{Children at home}

To reduce COVID-19 exposure, schools began to conduct learning remotely. As many childcare centers remained closed, this left many parents with the additional challenge of caring for children at home during the day. Providers appreciated that telehealth allowed them (and their clients) to engage with treatment while balancing childcare needs.

\section{Easing of regulatory restrictions on telehealth}

Many respondents credited the easing of telehealth regulations during COVID-19 with their ability to maintain their use of telehealth. The responses referenced COVID-era policy generally, as well as 
reimbursement policy specifically. Many of the providers referred to the use of telehealth as "completely dependent" upon the eased regulations becoming permanent. Some of the respondents expressed uncertainty about how long eased regulations would last, and several noted that the uncertainty "makes it difficult to do long-term planning/workflow development that includes [telehealth]."

\section{General telehealth themes}

The bulk of the verbatim responses shared general experiences with telehealth independent of the COVID19 pandemic. These 'general' responses reflected four subthemes: organizational conditions affecting ability to adopt telehealth, access, perceived treatment effectiveness, and cost-effectiveness. Within each of these subthemes, respondents detailed both advantages and disadvantages of using telehealth.

\section{Organizational conditions which affect ability to adopt telehealth}

A handful of respondents reported positive experiences with the integration of telehealth into their organizational workflow, stating that their organization had made telehealth easy to adopt. Others shared positive feedback about their organization's access to technology, which allowed them to easily download necessary platforms and navigate telehealth technology, even if they initially experienced a learning curve with setting up virtual services.

By contrast, multiple respondents reported struggles integrating telehealth into their organization's usual operations. Concerns about confidentiality, security, and encryption on platforms like Zoom were common. Several providers complained that they received limited support and training from management on adjusting to telehealth, that they were not given the equipment necessary for seamless and proper use of telehealth, and that managing multiple platforms was difficult. This difficulty was in some cases exacerbated by limited staff literacy. One respondent remarked that they struggled to adapt to telehealth at first, prompting them to "take online courses and webinars to learn [how to use telehealth]" on their own, as "It]he organization [doesn't] offer any webinars or trainings." Two others reported that co-workers were resorting to using HIPAA non-compliant platforms to treat patients due to lack of organizational infrastructure.

\section{Access}

Multiple providers remarked on client access to telehealth. Many respondents reported that telehealth removed barriers of access to transportation, distance to treatment, and access to childcare. As one provider put it, "No transportation - no problem! Gas is too expensive - no problem! No daycare - no problem!" 
Several respondents shared their perspective that virtual services opened the door for new populations to access treatment, such as clients with severe anxiety and clients who wish to avoid stigma associated with seeking treatment. Telehealth was also perceived as allowing respondents to provide remote treatment to clients residing in locations distant from them, such as clients in rural areas. As one respondent wrote: "[T]o have telephonic and telehealth end would mean many clients leaving services. Accessibility is everything, especially for the clients in the upper peninsula that we serve electronically right now. We'd love to see telehealth and telephonic appointment reimbursement continue. We've seen such an influx in SUD self-referrals because it is now so much more accessible." Some other providers observed that clients kept their telehealth appointments more often than they did with in-person appointments and that patients were generally more engaged in telehealth, with one provider noting that they "got better follow through and fewer no-shows." In addition, numerous respondents reported that their number of admitted patients increased during telehealth.

While some respondents perceived telehealth as opening up treatment, others perceived telehealth as making treatment less accessible. Several providers noted that switching to virtual services cut them off from some clients altogether, such as those who have unstable housing, lack adequate privacy for appointments, or refuse to use telehealth. One respondent expressed worry that not enough people in the rural community were aware of telehealth as a treatment option. Additional concerns were that clients were harder to reach, made fewer appointments over telehealth, disengaged from services, or missed more appointments. Providers also perceived that new clients took longer to engage in virtual services than their preexisting clients. Some respondents reported that their clients missed and rescheduled telehealth appointments more frequently than they did with in-person appointments, while others reported that several of their clients had fully disengaged from telehealth.

The most common concern about access to care was clients' limited access to technology. Many respondents complained that their clients did not have access to the internet, with a few citing poor broadband infrastructure in rural areas. Other providers reported that their clients did not have cell phones that could support telehealth platforms. Limited cell phone data and limited battery life were cited as problems, as sometimes clients would run out - or fear running out - of their cellular data or battery life if they used virtual services. In addition, respondents perceived some of their patients - such as clients from rural areas, low-income clients, cognitively impaired clients, and older adults - as more prone to having difficulty navigating technology. An overarching worry was that telehealth would exacerbate preexisting barriers to care, especially within historically marginalized patient populations and communities of color. Two respondents reported that technology issues raised barriers to continuous access and engagement during sessions, with one respondent adding that this situation posed unanticipated billing issues:

Cell phone quality \& connection problems ... can be a problem in meeting minimum session length requirements for billing. For example, I have a client whose phone frequently cuts out every 2 minutes. It gets frustrating for the client \& counselor to have to keep calling each other back. It also raises an ethical issue if we've only talked for 15 minutes but need to bill for a 25-minute session. If we don't bill for it, then 
it's as if the client wasn't seen for a session - not fair for the client to be penalized for having a poorquality phone, or for the program not to get credit for trying to serve the client despite the phone limitations.

\section{Perceived treatment effectiveness}

Another key subtheme pertained to the perceived effectiveness of telehealth. Respondents reported mixed opinions about whether telehealth improved or decreased the effectiveness of the care they were able to offer to clients. As an example, one provider wrote, "Truly a mixed bag. Works beautifully for some folks and want to continue even after COVID. For others, it is deeply frustrating and they long to come back face-to-face."

A handful of respondents reported that clients seemed more open or trusting over telehealth than during in-person sessions, while only respondents whose clients had preexisting trust issues reported lower trust levels. Providers also appreciated that clients' engagement in video care from home gave them insight into the client's home environment.

Several providers shared their perspective that telehealth was valuable if necessary but could never be an equally effective replacement for in-person treatment. "It is not an adequate substitute for in-person therapy," one respondent wrote, "but it is better than nothing." One of the key trepidations about effectiveness was missing important "body language cues" from clients during sessions. For example, one respondent noted, "without seeing them in person, it is hard to tell [when] they are struggling." Several others similarly felt that the "personal touch" was missing over telehealth, citing concerns with "rapport," "depth," and "connection," as well as experiences of "disconnect." One respondent shared that they were missing out on the "genuine transformation you see when the person comes to the office weekly." Other providers felt that the rigor of assessment was diminished over telehealth and that holding clients accountable was more difficult.

\section{Cost-effectiveness}

Another subtheme pertained to perspectives of the cost-effectiveness of telehealth. These perspectives were mixed. Some respondents felt that telehealth was more productive and efficient. One respondent commented that "the more I use it, the more I like it because it saves me time, money, and energy." Another said that "administratively it is cost effective because I do not have staff with as much 'windshield time' as they drive across 4 rural counties to provide services." Others said that telehealth required a greater investment of work and time, citing technology setup and building rapport with clients.

\section{Recommendations to continue telehealth delivery}


Three subthemes emerged related to recommendations to advance telehealth: continuing to ease or change telehealth restrictions, investing in broadband infrastructure and institutional support, and combining telehealth and face-to-face intervention.

\section{Continued easing of regulations}

The most frequent suggestion was to continue telehealth reimbursement and relaxed regulations. "It would be a shame," one respondent said, "to again implement the limitations." Beyond continuation of eased regulations, some respondents took it a step further and advocated for changed regulations. One respondent suggested, "Let's change the previous regulations to facilitate the use of telehealth by nonlicensed professionals such as recovery coaches, case managers and counselors." Another echoed this, saying that "we need to continue to allow bachelor level and recovery support services staff to use telehealth post-pandemic."

\section{Investing in broadband infrastructure and institutional support}

Another popular suggestion was to invest in expanding access to infrastructure in rural areas to increase access to telehealth services. "Internet service providers need to MAJORLY step up availability and affordability of internet services in rural areas," one respondent said. Meanwhile, another provider noted that ensuring equitable access to care would require large-scale changes. "These barriers," they said, "are too large for our non-profit agency to address." Institutional support was also viewed as critical. Multiple providers expressed a desire for more training, technical support, and equipment for staff, as well as increased security and improvement of telehealth platforms.

\section{Combining telehealth and in-person care}

Finally, several respondents explained that they intended to continue using a combination of telehealth and in-person care after the end of the COVID-19 pandemic. Many providers shared their perspective that although they do not prefer telehealth to in-person care, it should continue as an option for clients.

However, it was commonly acknowledged that the ability to offer a hybrid combined model would depend on reimbursement. As an example, one respondent wanted their care model to integrate telehealth, but explained that "without reimbursement ability, it is not an option at all."

One respondent took it a step further and argued that telehealth should be viewed as a key part of the future of treatment, explaining: "I think it's time to not see telehealth only as an alternative in times of urgent need, but rather as a necessity that requires thorough training, development of sound policies and implementation of secure telehealth systems." 


\section{Discussion}

This study advances our knowledge of how the behavioral health workforce experiences telehealth delivery. Results revealed three key emergent themes: COVID-specific issues with telehealth, general issues with telehealth, and future directions for telehealth. Across these themes, key issues pertained to access to care, the effectiveness and cost-effectiveness of treatment, and the need for institutional support.

Although multiple hurdles to effective and accessible use of telehealth remain, telehealth has proven to be an invaluable and convenient tool for SUD and MH treatment providers, even after COVID-19 public safety precautions subside. Respondents overwhelmingly praised that telehealth addresses the barriers of long distances to treatment and access to transportation. Lack of commute is often cited as a benefit to telehealth, as was largely echoed in these results $[12,13]$. Telehealth has been noted for its ability to provide access to harder-to-reach populations and was originally created for this purpose [13]. It is notable that even when these providers began to use telehealth for a reason other than initiating deliberate outreach to harder-to-reach demographics, some still observed new clients entering treatment.

Among respondents raising barriers to telehealth, concerns about clients' access to technology were resoundingly common. Notable in this study is the frequency with which respondents complained of the following technology barriers specific to clients' cellular devices: devices incapable of supporting telehealth software, limited cell phone data or battery life (and clients' resulting caution not to waste these), and clients not owning a cellular device. Other technology issues, such as older adults' difficulty with technology and limited broadband access in rural or otherwise underserved areas, have been noted before as barriers to telehealth [13]. Access to technology is a particular concern in treatment for SUD, which affects those experiencing homelessness and poverty disproportionately [14, 15].

When considering future directions, many of the respondents explicitly supported a hybrid model of treatment combining virtual and in-person treatment. One option to apply such a model is to enable the provider and client to collaborate to choose the best treatment modality. This hybrid treatment model has been recognized as a way forward as post-COVID-19 public safety precautions subside $[16,17]$.

Another common theme related to the future of telehealth delivery was the need to continue easing regulatory restrictions on telehealth. Many respondents felt that their ability to continue offering virtual services hinged upon whether COVID-19-era eased reimbursement policy would continue. Respondents' concern about reimbursement is consistent with other research that has been conducted on providers' attitudes about telehealth during the COVID-19 pandemic: Guinart et al.'s [7] evaluation of the 2021 survey of $\mathrm{MH}$ care providers concludes that "all stakeholders involved should ensure that the current reimbursement flexibility related to the COVID-19 pandemic remains in effect after the outbreak has ended." Several others have endorsed continued relaxation of reimbursement regulations [18].

Respondents' attitudes toward the effectiveness of virtual services mirrors findings of past research: A survey administered to nearly 1,500 MH professionals in May and June 2020 found that while 
respondents endorsed telehealth as important, necessary, and effective, providers gave a relatively lower endorsement of the belief that telehealth is as effective as in-person care [19]. Pre-COVID-era studies comparing in-person care outcomes with telehealth care outcomes have found them to be comparable [19-21].

\section{Limitations}

Results of this study should be considered in the context of several limitations. Most notably, the question to which respondents responded was extremely open-ended. Thus, this should perhaps be viewed as an exploratory study. In addition, a convenience sample was used for the original survey, consisting of individuals who utilize MHTTC and ATTC services. The respondents from the SUD survey represented only 33 states and Puerto Rico; those from the MH survey represented only 25 states, the District of Columbia, Puerto Rico, and the U.S. Virgin Islands. Moreover, the current sample consisted of only those who were willing to answer the open-ended question. The reasons motivating respondents' choice to respond to this question are unknown. The experiences and perceptions of those who volunteered responses may differ from those who did not respond. Survey respondents may also have differed in their understanding of the purpose of the question (i.e., whether it was intended to improve telehealth platforms, for research purposes, etc.). These factors may contribute to selection bias and compromise the generalizability of the findings.

\section{Conclusions}

As noted in prior research on providers' attitudes towards telehealth, further longitudinal research comparing treatment outcomes of those receiving in-person and virtual services will be necessary to undergird organizations' financial support, and perhaps also legislative support, of virtual SUD and MH services. Future research must compare the outcomes of in-person and telehealth treatment performed during the COVID-19 pandemic.

\section{Abbreviations}

ATTC: Addiction Technology Transfer Centers

COVID-19: Novel coronavirus 2019

$\mathrm{MH}:$ Mental health

MHTTC: Mental Health Technology Transfer Centers

SUD: Substance use disorder

\section{Declarations}




\section{Ethics approval and consent to participate}

The University of Wisconsin Health Sciences Minimal Risk Institutional Review Board deemed this research as being exempt human subjects research as defined under 45 CFR 46 , thus waiving the need for participant consent (ID\#: 2020-0551). Despite this exemption, all participants were nonetheless informed that the survey was voluntary in the email solicitation to complete the survey. All methods were carried out in accordance with the relevant guidelines and regulations.

\section{Consent for publication}

This is not applicable as there are no personal identifiers present in the dataset.

\section{Availability of data and materials}

The datasets used and/or analyzed during the current study are available from the corresponding author on reasonable request.

\section{Competing interests}

Author Molfenter has $<1 \%$ stock ownership in CHESS Mobile Health. CHESS Mobile Health does not provide or facilitate telephonic or video care delivery. Dr. Molfenter has worked extensively with his institution to manage any conflicts of interest. No other authors have competing interests regarding this manuscript.

\section{Funding}

This manuscript was funded by the following Substance Use and Mental Health Administration (SAMHSA) Addiction Technology Transfer Center (ATTC) cooperative agreements:

UR1TI080209, UR1TI082504, UR1T1080215, UR1TI080207, UR1TI080203, UR1T1080200, UR1TI080201.

\section{Authors' contributions}

AK coded the data, analyzed the data, and drafted the manuscript. SB reviewed the coding framework and substantially revised the manuscript. NJ provided guidance for the coding framework and the interpretation of the data. TM provided oversight for this study and assisted in designing the coding framework and revisions to the manuscript. AA, MC, DG, BH, NJ, and ST helped to steer the direction of the analysis. MC, DG, BH, NJ, and ST also reviewed and revised the manuscript. 


\section{Acknowledgements}

The study team gratefully acknowledges the editorial assistance from Maureen Fitzgerald and Judith Ganch.

\section{References}

1. Molfenter T, Roget N, Chaple M, BehIman S, Cody O, Hartzler B, et al. Use of telehealth in substance use disorder services during and after COVID-19: online survey study. JMIR Ment Health. 2021;8(2):e25835.

2. Molfenter T, Heitkamp T, Murphy AA, Tapscott S, Behlman S, Cody OJ. Use of telehealth in mental health (MH) services during and after COVID-19. Community Ment Health J. 2021:1-8.

3. Wosik J, Fudim M, Cameron B, Gellad ZF, Cho A, Phinney D, et al. Telehealth transformation: COVID19 and the rise of virtual care. J Am Med Inform Assoc. 2020;27(6):957-62.

4. Huskamp HA, Busch AB, Souza J, Uscher-Pines L, Rose S, Wilcock A, et al. How is telemedicine being used in opioid and other substance use disorder treatment? Health Aff (Millwood). 2018;37(12):1940-7.

5. Substance Abuse and Mental Health Services Administration (SAMHSA). FAQs: Provision of methadone and buprenorphine for the treatment of Opioid Use Disorder in the COVID-19 emergency. Rockville, MD: Substance Abuse and Mental Health Services Administration; 2020 April 21. Available from: https://www.samhsa.gov/sites/default/files/faqs-for-oud-prescribing-and-dispensing.pdf.

6. Haque SN. Telehealth beyond COVID-19. Psychiatr Serv. 2021;72(1):100-3.

7. Guinart D, Marcy P, Hauser M, Dwyer M, Kane JM. Mental health care providers' attitudes toward telepsychiatry: a systemwide, multisite survey during the COVID-19 pandemic. Psychiatr Serv. 2021;72(6):704-7.

8. Brooks E, Turvey C, Augusterfer EF. Provider barriers to telemental health: obstacles overcome, obstacles remaining. Telemed J E Health. 2013;19(6):433-7.

9. Cowan KE, McKean AJ, Gentry MT, Hilty DM. Barriers to use of telepsychiatry: clinicians as gatekeepers. Mayo Clin Proc. 2019;94(12):2510-23.

10. Turner Lee N, Karsten J, Roberts J. Removing regulatory barriers to telehealth before and after COVID19. The Brookings Institution and The John Locke Foundation; 2020 May. Available from: https://www.brookings.edu/wp-content/uploads/2020/05/Removing-barriers-to-telehealth-beforeand-after-COVID-19_PDF.pdf.

11. Hsieh H-F, Shannon SE. Three approaches to qualitative content analysis. Qual Health Res. 2005;15(9):1277-88.

12. Gajarawala SN, Pelkowski JN. Telehealth benefits and barriers. J Nurse Pract. 2021;17(2):218-21.

13. Steidtmann D, McBride S, Mishkind MC. Experiences of mental health clinicians and staff in rapidly converting to full-time telemental health and work from home during the COVID-19 pandemic. 
Telemed J E Health. 2021;27(7):785-91.

14. Midboe AM, Byrne T, Smelson D, Jasuja G, Mclnnes K, Troszak LK. The opioid epidemic in veterans who were homeless or unstably housed. Health Aff (Millwood). 2019;38(8):1289-97.

15. Shakir M, Wakeman S. Substance use disorder and telemedicine: opportunity and concern for the future. J Gen Intern Med. 2021;36(9):2823-4.

16. Balcombe L, De Leo D. An integrated blueprint for digital mental health services amidst COVID-19. JMIR Ment Health. 2020;7(7):e21718.

17. Smith K, Ostinelli E, Macdonald O, Cipriani A. COVID-19 and telepsychiatry: development of evidencebased guidance for clinicians. JMIR Ment Health. 2020;7(8):e21108.

18. Kannarkat JT, Smith NN, McLeod-Bryant SA. Mobilization of telepsychiatry in response to COVID-19moving toward 21(st) century access to care. Adm Policy Ment Health. 2020;47(4):489-91.

19. Shigekawa E, Fix M, Corbett G, Roby DH, Coffman J. The current state of telehealth evidence: a rapid review. Health Aff (Millwood). 2018;37(12):1975-82.

20. Hilty DM, Ferrer DC, Parish MB, Johnston B, Callahan EJ, Yellowlees PM. The effectiveness of telemental health: a 2013 review. Telemed J E Health. 2013;19(6):444-54.

21. Lazur B, Sobolik L, King V. Telebehavioral health: an effective alternative to in-person care. Issue Brief. New York City: Milbank Memorial Fund; 2020 October. Available from: https://www.milbank.org/wp-content/uploads/2020/10/TeleBH_B_6.pdf. 